\title{
Design of a Comprehensive Condition Monitoring System for Gas Turbine Engines
}

\author{
David May \\ Mechanical Engineering, \\ Concordia University \\ davidmay44@hotmail.com \\ Chien Yu Chen \\ Mechanical Engineering, \\ Concordia University \\ yako_c@hotmail.com
}

\author{
Steven Farber \\ Mechanical Engineering, \\ Concordia University \\ sfarber@me.concordia.ca \\ Dr. Ion Stiharu
Mechanical Engineering,
Concordia University
istih@vax2.concordia.ca
}

\author{
Vincient Poirier \\ Mechanical Engineering, \\ Concordia University \\ vpoirier@ciadi.concordia.ca
}

\begin{abstract}
This report contains the work of the capstone design team, assigned to design a comprehensive, cost effective, monitoring system for gas turbine engines. The main objective of the design was to produce a system capable of detecting and recording only special conditions or events. As a sample engine, the SR-30 gas turbine was used. A simulation of the engine using GSP software provided a model. The model was used to determine the healthy operation of the engine. The integration of a developed algorithm has enabled the system to be used for aerodynamic component monitoring, as well as, mechanical systems monitoring.
\end{abstract}

\section{Introduction}

Gas turbine engines constitute a complex system, requiring adequate monitoring to ensure safety and costefficient maintenance. Companies are constantly striving for ways to improve engine performance while simultaneously trying to reduce cost. In order to reduce a high number of incidents and maintenance costs, enginemonitoring systems have become an integral part of the modern gas turbine. The Society of Automotive Engineers (SAE) published the first Aircraft Gas Turbine Engine Monitoring System guide in 1981[1].

The multi-disciplinary nature of the project, a fusion of Aerodynamics, Structure, System Modeling and Computer Engineering makes the design and implementation particularly complicated. There was a constant trade-off between feasibility and functionality.

The development of a useful monitoring system requires that the engineer decide on the key parameters that affect performance, and further, it requires their predictive (or threshold) values for the given conditions.
The actual inputs to the component (as recorded by the sensors) and the performance parameter data (postprocessing), can then be compared to the predictive or threshold value to obtain a deviation from predicted results.

Based on the above discusion, data from a gas turbine engine is required. The cost effective alternative to operating a gas turbine engine was to build a computer simulation of the engine using component characteristics.

Efficient data storage was also a prime objective of the design. This was achieved with an event driven architecture. The system does not record data continuously. Data is sampled and processed, suited to the type of information being recorded. The data storage device of choice is the SmartCard system. The design objectives were a low cost, event driven philosophy and SmartCard based data storage. The SmartCard sender/receiver unit is interfaced to the sensors with the use of a micro-controller.

\section{Simulation of a Gas Turbine Engine}

Performance data is needed to determining healthy engine operation. Once the design and off-design performance is known for an engine, a simulation could be created using software for simulating typical engine missions. Temperature, pressure, and other key performance parameters could be obtained from the simulation, and then used as the threshold values of the engines healthy operation to which the actual measured values could be compared.

A survey of gas turbine software was conducted to investigate options for simulating a gas turbine engine. The simulation tool to suit our needs would be required to be able to perform 0-D modeling of design and off- 
design engine performance using the characteristic performance curves of the engine. The gas turbine software survey led to the discovery of Gas Turbine Simulation Program (GSP), which was available through the National Aerospace Laboratories.

\subsection{SR-30 Mini-Lab Gas Turbine}

One gas turbine engine that was accessible with design point data, available in open source, was the SR30 Mini-Lab gas turbine engine developed by Turbine Technologies (Figure 1). The SR-30 is designed for academia to teach the principles of gas turbine operation. The engine is designed to be representative of a turbojet engine of a larger scale [2].

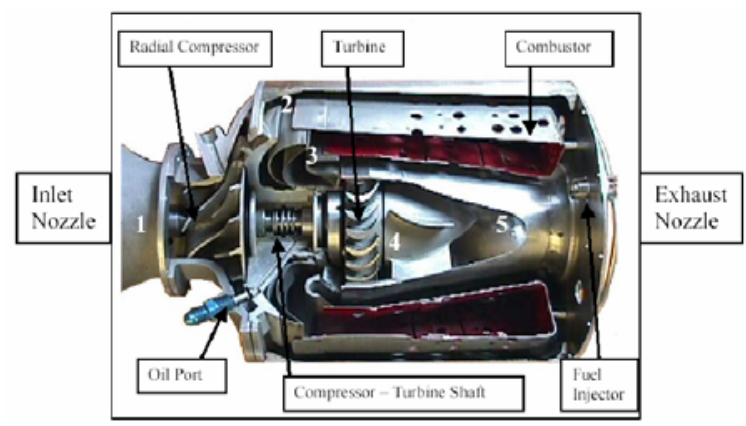

Figure 1. SR-30 engine components [3]

\subsection{Generating the Gas Turbine Component Maps}

A gas turbine is designed for minimum pressure losses at the design point. It is also important that the engine performs with moderate losses at off-design conditions. The prediction of the off-design performance can be calculated through loss correlations which are found in open source.

A design point analysis of the SR-30 was required in order to analytically build the component maps of the turbine and compressor. The University of Minnesota published a paper which contains blade geometry and an approximate design point analysis [3]. For each component, the design point velocity triangles are drawn and the published results are verified. From this point on we could apply the analytical analysis and design the component performance maps to be inputted into GSP.

\subsection{Calculating the Turbine Map}

The SR-30 has a single stage turbine composed of one row of nozzles and one rotor. The published design point losses were calculated through Ainley and Mathieson correlations [4]. The results are reasonable and matched our verification.

The off-design velocity triangles were built, first by taking the inlet flow gas angle and deviation across the nozzle as constant at all off-design conditions. This results in the losses across the nozzle being constant for all off-design conditions. Next, by sweeping through values of spool rotational speed and mass flow we drew velocity triangles for the rotor. Figure 2 demonstrates the principles of varying the incidence at off-design conditions across the turbine rotor blades.

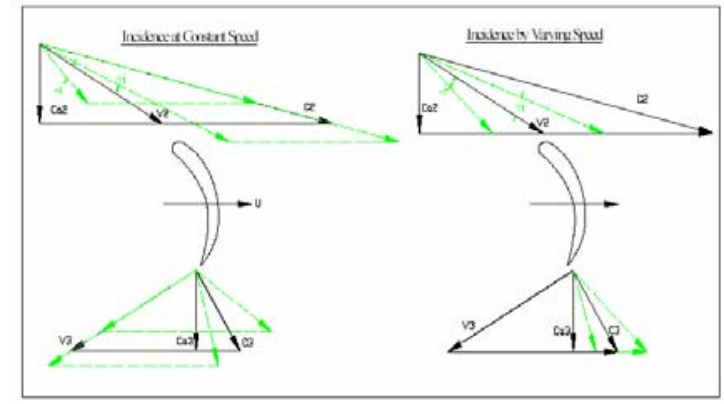

Figure 2. Incidence applied to velocity triangles using standard turbine notation

A spreadsheet in Excel was created for performing the iterative calculations of the velocity triangles. The loss correlations that were chosen for the off-design analyses are from Ainley/Mathieson/Dunham/Came (AMDC) [4]. The stalling incidence of the rotor blades was found to occur at an incidence of $+11^{\circ}$. The Secondary losses are taken to be constant at values of incidence outside of the range of $-16.5^{\circ} \leq \mathrm{i} / \mathrm{i}_{\text {stall }} \leq 11^{\circ}$ as predicted by AMDC. For each off-design point, the inlet temperature and pressure was taken at the design conditions of $233.5 \mathrm{kpa}$ and $973^{\circ} \mathrm{K}$. Off-design data was calculated for several constant speed lines in Excel for equal increments of stage pressure ratio. The turbine performance map was then put into GSP format to be used in the software (Figure 3).

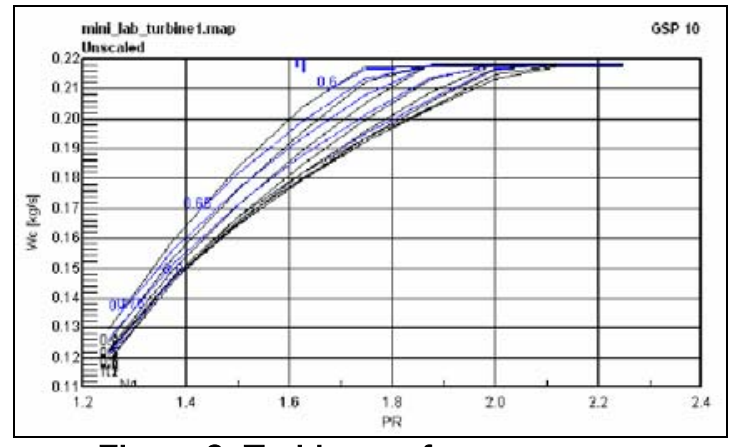

Figure 3. Turbine performance map

\subsection{Calculating the Compressor Map}

The SR-30 is equipped with a centrifugal compressor with both a vaneless and vaned diffuser. All design point information, taken from reference 3 , was verified and found to be reasonable for the engine. 
The off-design calculations of a centrifugal compressure is iterative requiring a guess of the density at the exit of the impeller and then checking the guessed value with the value obtained from the loss calculations. The correlations used for the compressor losses were taken from a published paper which states the optimum set of loss correlations for centrifugal compressors which can be found in open source [5]. Excel was once again used for performing the calculations and all iterations. The assumptions made in the off-design calculations are that the absolute inlet velocity remains axial, and the design point value of the slip factor was also relatively constant for all off-design conditions. Figure 4, shows how the off-design incidence was applied to the impellor for varying and constant rotational shaft speeds.

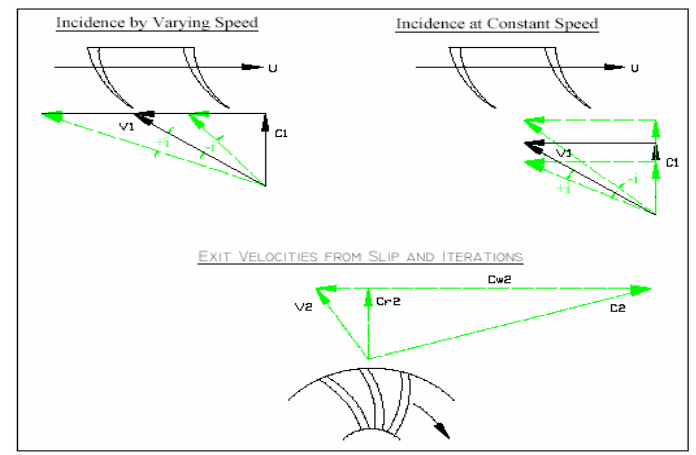

Figure 4. Incidence applied to velocity triangles using standard compressor notation

The compressor performance map could be built with only a few values of incidence for different constant shaft speed values. The program Smooth-C was used to plot the compressor performance map (Figure 5). Smooth- $C$ is a tool that produces high quality compressor characteristics from the input data.

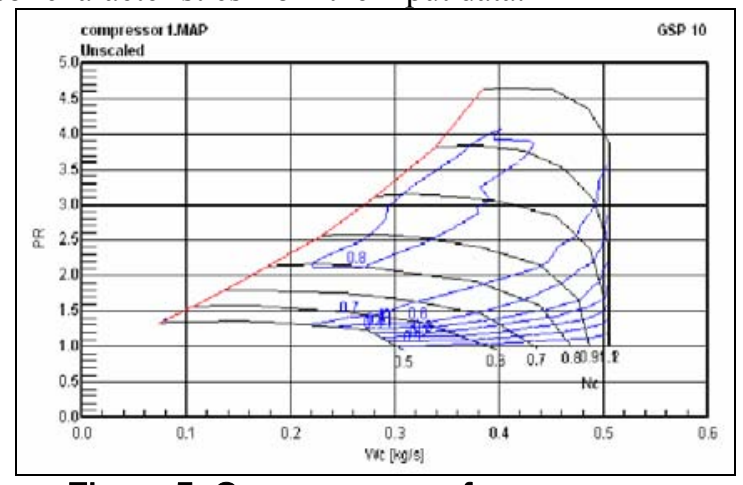

Figure 5. Compressor performance map

\subsection{GSP Model}

The SR-30 was simulated in GSP using gas path standard components and a manual fuel controller. The block diagram of the engine is shown in Figure 6.

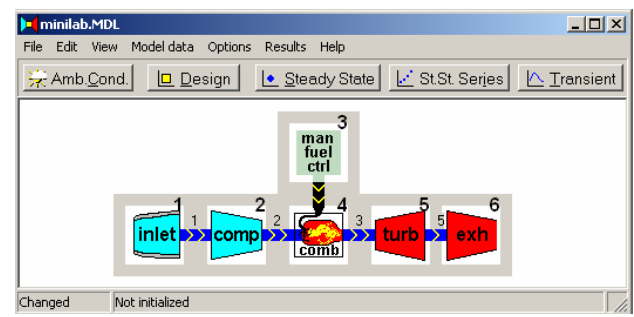

Figure 6. GSP model of the SR-30 engine

The component maps were integrated into the model along with all design point information. Figure 7, shows the experimental and simulated results together. These results show that the GSP model of the SR-30 can be used to accurately predict the performance of the engine to within $7 \%$ error.

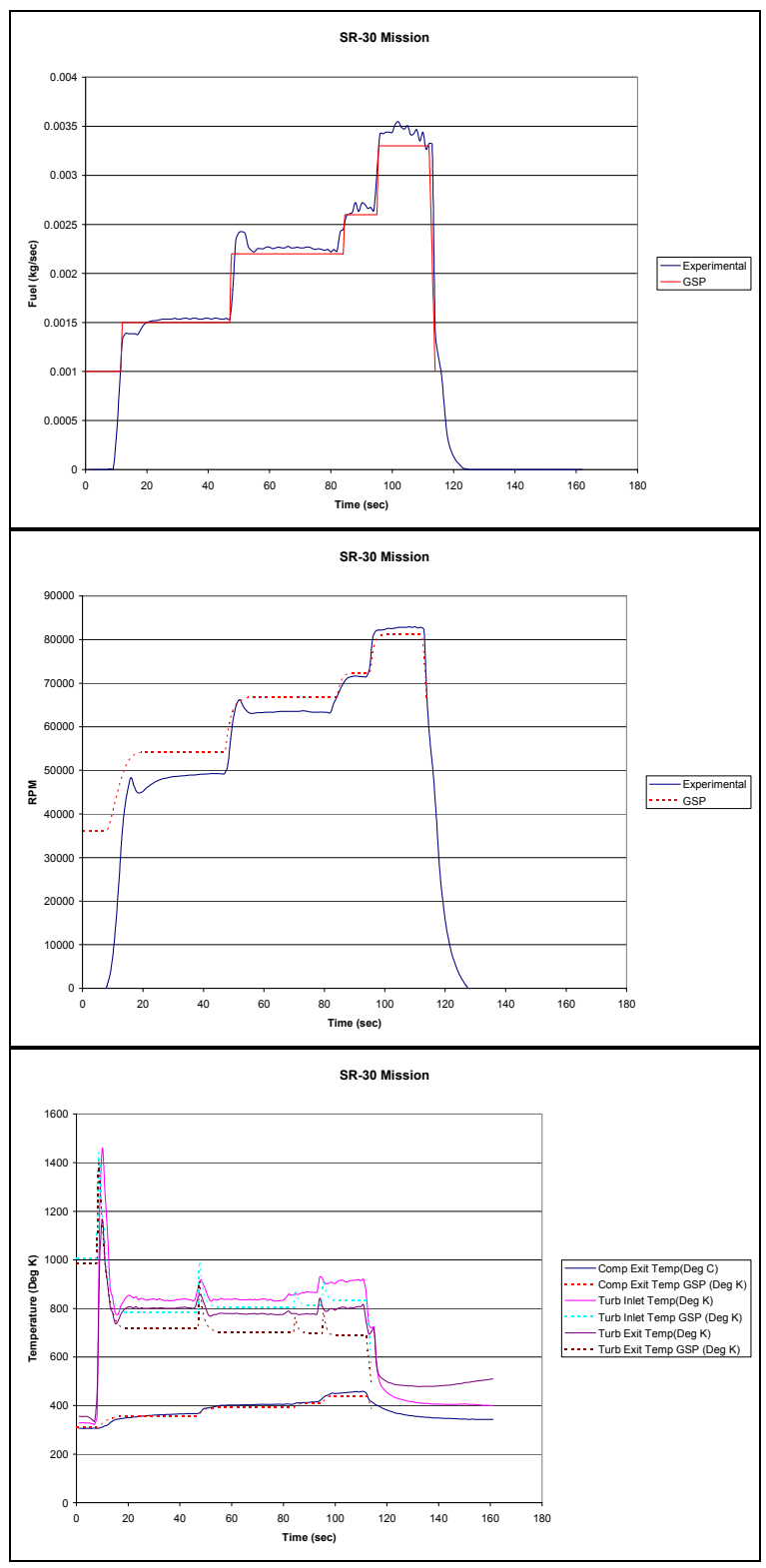




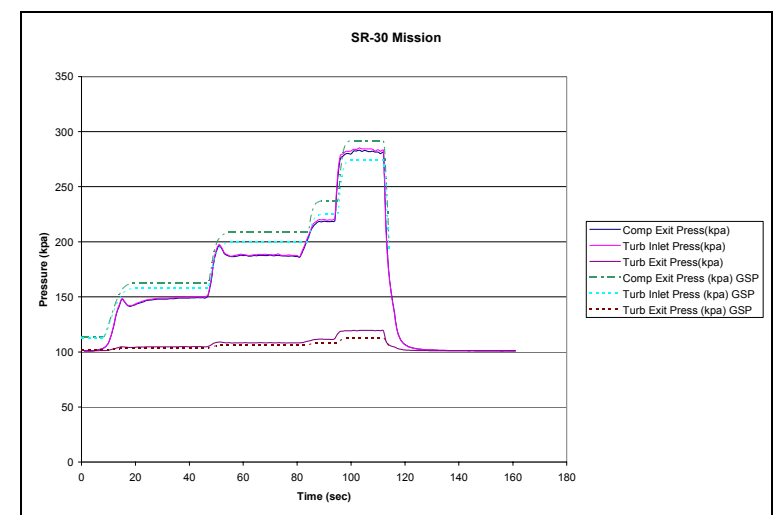

Figure 7. Comparison of experimental and GSP results from a mission

\subsection{Determining the Threshold Values}

The objective of obtaining the GSP model is to determine the healthy operation of the engine. Because a gas turbine engine will deteriorate over time, a range of acceptable operating values needed to be determined. Engine deterioration was simulated through GSP by selecting deterioration in the component properties. With acceptable values of engine deterioration determined, which would be the limits to the definition of healthy engine operation, missions could then be simulated and healthy parameters are determined.

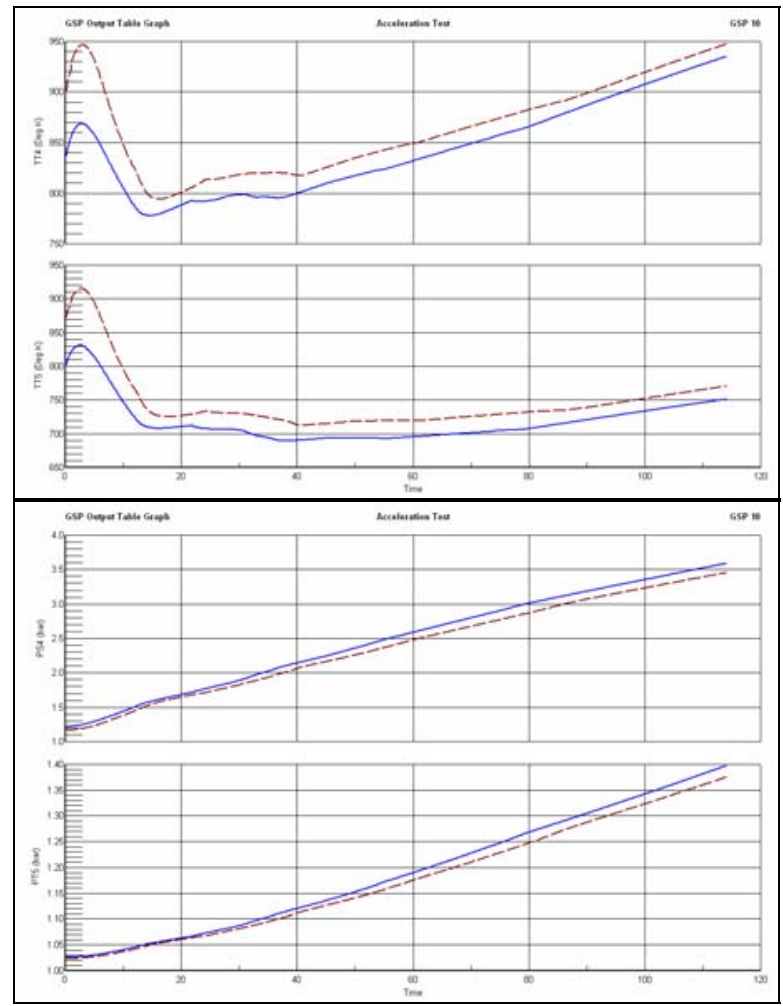

Figure 8. A mission with zero deterioration [solid line] and deterioration [dotted line]
A sample mission performed using GSP simulated an acceleration of the SR-30 engine from an idle fuel flow of $0.001 \mathrm{~kg} / \mathrm{s}$ to $0.005 \mathrm{~kg} / \mathrm{s}$ over 114 seconds. The same mission was again run with a turbine deterioration consisting of $+1 \%$ increase in corrected mass flow and $-2 \%$ drop in efficiency (Figure 8). Results like these could then be used as healthy limits in the monitoring system.

\section{An Aerodynamic Component Monitoring System.}

There is a distinction between two types of monitoring undergone in the system, component performance monitoring and component failure monitoring. The distinction is made based on the objective of the monitoring algorithm.

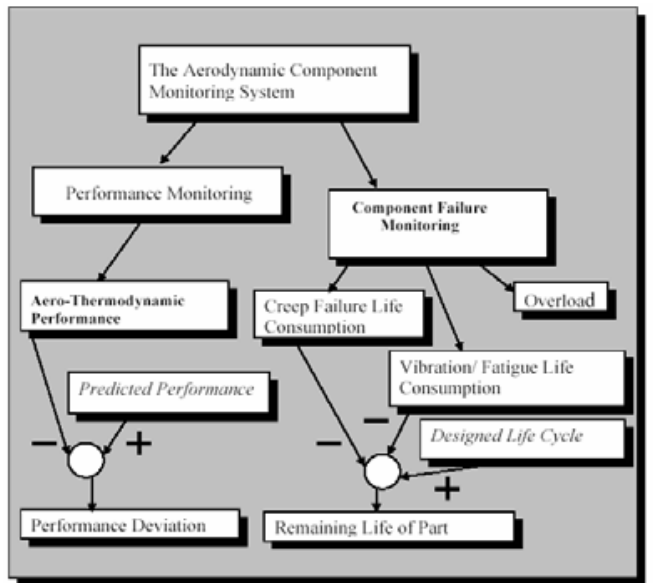

Figure 9. The aerodynamic component monitoring architecture

Figure 9, displays the "generic" monitoring algorithm of an aerodynamic component for the monitoring system being discussed. Any given component will generally be affected by each of the parameters. However, in some cases the effect of any one of these parameters may be determined to be negligible and therefore the model can be simplified. Additionally, certain components may require special attention and modification. However, in the vast majority of cases, it is believed that the model presented is sufficiently robust.

\subsection{Parameters of Importance}

The typical parameters that are recommended for monitoring in aircrafts are:

1) Temperatures (inlet, outside air, exhaust gas, compressor, turbine, bleed air)

2) Pressures (inlet, compressor, discharge, lube oil, bleed air) 
3) Oil system (quantity, filters, consumption, debris, contamination)

4) Vibration (rotors, shafts, afterburners, reduction gears, bearings, transmissions, and accessories)

5) Life usage (operating hours, start times, fatigue, stresses, cracks)

Additional parameters such as speeds, fuel flow, throttle position, nozzle position, and stator position are also of interest. [6,7]

\subsection{Performance Monitoring}

Component performance monitoring seeks to obtain measurement of the real time aerodynamic performance of the component and to compare these measured values to predicted values of performance. The comparison of these two values results in a deviation from predicted performance.

\subsubsection{Aerodynamic Component Performance Monitoring}

Performance deterioration of aerodynamic components in a gas turbine engine is always detrimental to the efficiency of the gas turbine as a whole. That is why its monitoring is interesting and desirable. The algorithm presented below is specifically applicable to the compressor stage of a gas turbine. Performance monitoring of the compressor stage is particularly interesting because its performance is greatly affected by off-design conditions.

It was decided that the two main performance characteristics to monitor would be pressure ratio and efficiency. The basic doctrine is this; take the measured values of performance, compare these with predictive values, and output these parameters in the form of dimensionless percent drop in performance.

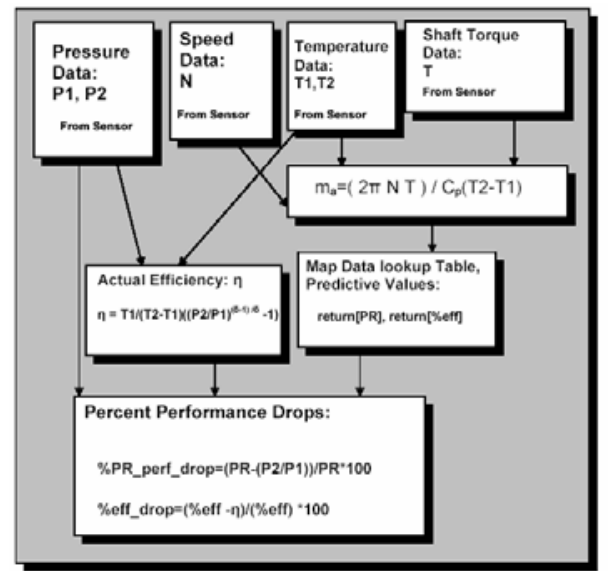

Figure 10. The aerodynamic performance algorithm
As is demonstrated in the Figure 10, standard sensor inputs such pressure, temperature, and rotational speed are utilized. In addition to these, a torque sensor would give us just the required information necessary to calculate the mass flow rate though the system.

Once mass flow rate is calculated in the microcontroller this information, along with the rotational speed of the shaft, can be used to obtain predictive values of efficiency and pressure ratio. These predictive values are obtained by the micro-controller in a way analogous to somebody reading a performance map that has been compiled through experiment or aero-thermodynamic calculations (as was done in this study). The graph is stored in the micro-controller in the form of a lookup-table; for any given values of rotational speed and mass flow rate, values of pressure ratio and efficiency can be obtained.

Upon obtaining these predicted parameters they may be compared with the actual values of efficiency and pressure ratio. Actual efficiency is calculated as shown in the block diagram. A value of deviation from predicted values may be saved to the SmartCard receiver in the form of a percent drop, or it may be displayed to a screen.

The compressor is given as an example that may be modified for use in monitoring other aerodynamic components. However, the appropriate sensor data is necessary. Also, it must be noted that predictive data must be available for comparison.

\subsection{Aerodynamic Component Failure Monitoring}

By contrast, component failure monitoring has as its objective the monitoring of the usage of a component during any given mission, and then the subtraction of this usage from its designed life cycle. The difference between these two values is the remaining life to failure.

\subsubsection{Life Monitoring of Components That Fail Due to Creep}

Aerodynamic component failure in a gas turbine engine is often affected by creep. The mechanism of creep is influenced by temperature and stress. The high temperatures of the gas path produce the first of the two requirements, and centrifugal stress from shaft rotation produces the second (see Figure 11 and relations presented). If parts susceptible to this mode of failure go unmaintained, this will eventually result in catastrophic failure. That is why some form of monitoring is desirable and required. The algorithm presented below is specifically applicable to the turbine stage of a gas turbine. Failure monitoring, of the turbine stage, is particularly interesting because it is very susceptible to 
failure. The mal performance of the turbine stage is detrimental to the entire engine.

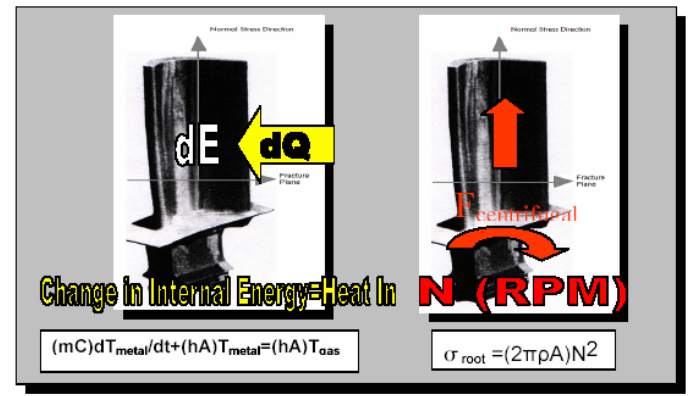

Figure 11. Mechanism of creep in a gas turbine

Failure Monitoring is carried out as follows; obtain a measured value of component usage during any given mission and subtract this from the previously recorded values of remaining component life. Finally, obtain a dimensionless value of percent usage until maintenance is required.

As the algorithm demonstrates in Figure 13, standard sensor inputs of local gas temperature and rotational speed are used.

Two values must be calculated in the microcontroller with this information, Part Stress and Part (Metal) Temperature. This is done using the relations presented (see Figure 12 for graphical example of the calculation of Metal Temperature from gas temperature).

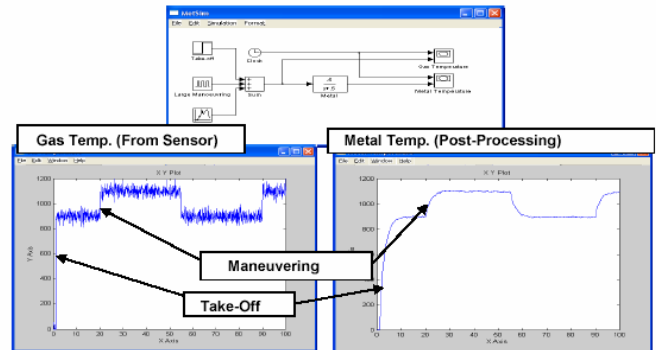

Figure 12. Calculation of metal temperature from gas temperature

These values can be used to obtain predictive values of time until failure at the given conditions. These predictive values are obtained by the micro-controller in a way analogous to somebody reading a Larsen-Miller chart that has been compiled through experiment for the given component material. The graph is stored in the micro-controller in the form of a lookup-table; for any given values of Part Stress and Metal Temperature, values of part life can be obtained.

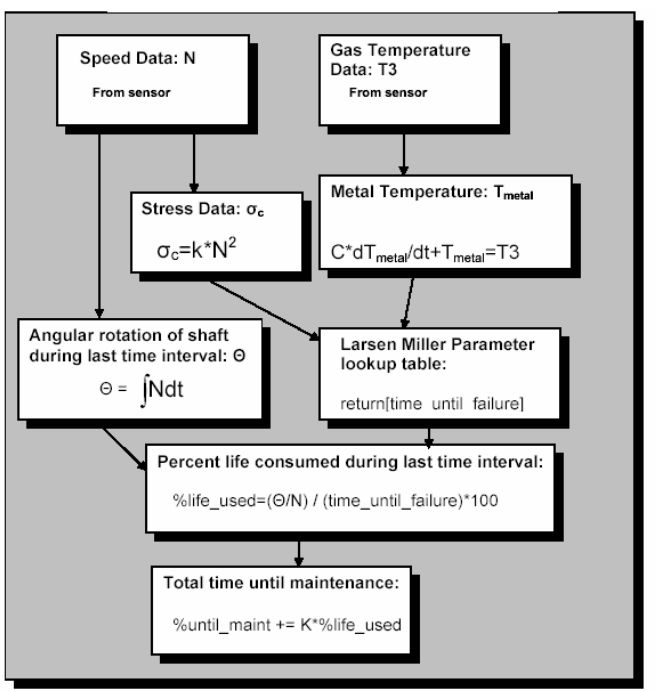

Figure 13. Algorithm for monitoring creep

Upon obtaining the time until failure, at the given condition, and with knowledge of the time, for which it was operating at these conditions, a value of fraction of life used (fl=time/time until failure) may be obtained. It may be noted that this is a modified form of the Palmgren-Miner Rule. It is assumed that once,

$$
f=f 1+f 2+f 3+\ldots+f n=1
$$

the part will fail and will surely require maintenance. Since we would like to maintain the engine, and not wait for the part to fail we can simply multiply these fractions by some constant $k$ such that,

$$
f=k^{*} f 1+k^{*} f 2+k^{*} f 3+\ldots+k^{*} f n=1
$$

before failure and will send a flag to the SmartCard indicating maintenance is required. For Example: $k=10$ will send a maintenance flag every time one tenth of the part life is used. This is shown in the Figure 14.

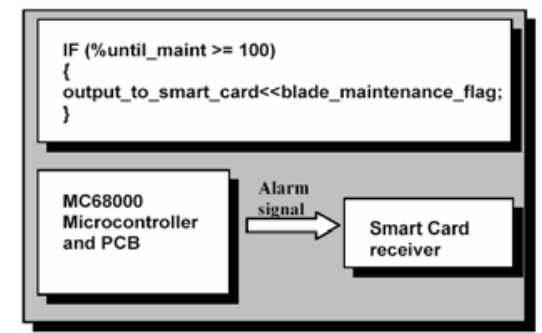

Figure 14. Generation of alarm signal

\subsubsection{Life Monitoring of Components That Fail Due to Fatigue}

Due to the nature of the operating environment of a gas turbine engine, aerodynamic components are constantly subjected to repeated loads, also called cyclic loads. Despite stresses below the material's ultimate strength, dynamic structural deformation over time leads to development of cracks that ultimately cause the 
failure of the material. This type of damage due to cyclic loading is termed "fatigue".

The state of stress created in a turbine blade comes from two principle factors. The torque created by the turning (deflection) of the gas as it passes through the turbine blade passages, in combination with the centrifugal stresses produced by the rotation of the shaft, creates a very complex asynchronous, two dimensional stress field.

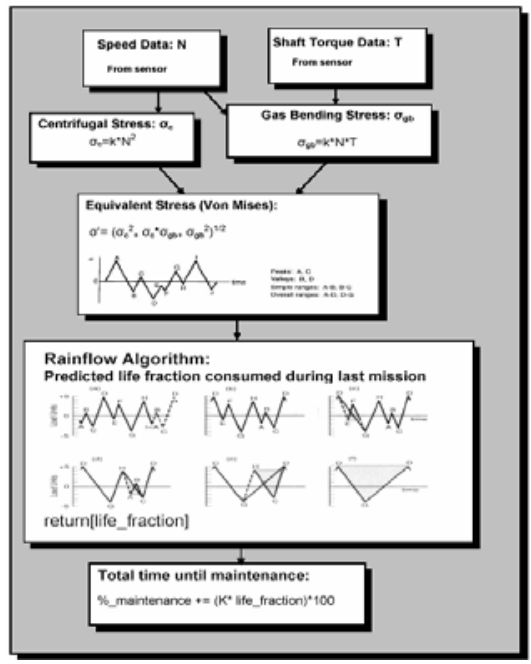

Figure 15. Fatigue algorithm for aerodynamic components

As shown in Figure 15, the gas bending stress is directly proportional to change in tangential velocity of the gas, and as a result, it is proportional to work across the stage. Also as explained previously the centrifugal stress is proportional to the speed squared. These two principle stresses (centrifugal and gas bending) can be combined via the Von Mises equivalent stress.

In cases of highly irregular loading the best approach is to use is the rainflow method. Irregular loading is characterized by a series of peaks and valleys, which occurs when stresses change direction. The rainflow method is an algorithm that identifies and counts peaks and valleys.

The data obtained will be used in a modified Goodman Diagram (MGD) to obtain the number of cycles to failure $\left(\mathrm{N}_{\mathrm{f}}\right)$ for each loading combination. Those local cycles to failure will be converted as fraction of total component life. The component fails when the sum reaches 1 .

$$
\frac{N_{1}}{N_{f 1}}+\frac{N_{2}}{N_{f 2}}+\frac{N_{3}}{N_{f 3}}+\ldots=\sum \frac{N_{n}}{N_{n f}}=1
$$

As we did for the creep algorithm, we would like to maintain the engine, and not wait for the part to fail. One can simply multiply these fractions by some constant $K$ such that,

$$
\sum K \times \frac{N_{n}}{N_{n f}}=1
$$

before failure and will send a flag to the SmartCard indicating maintenance is required. For Example: $\mathrm{K}=10$ will send a maintenance flag every time one tenth of the part life is used.

\section{Mechanical Systems}

Periodic measurement of vibration characteristics of the machinery and structures is essential to ensure safety margins while reducing maintenance costs. Any observed shift in the natural frequencies or other vibration characteristics will indicate either a failure or a need for maintenance $[8,9]$.

The life of a machine follows a classic bathtub curve. Initially the vibration level decreases during the "breaking-in" period of the machine and then will slowly increase with use due to wear of the components. This is then followed by a sudden increase in vibration due to excessive wear until failure or breakdown [10]. The condition based maintenance scheme uses fixed interval measurements to monitor the variation of vibrations in both transient and cruise running conditions.

A means to determine the health of the mechanical systems of the engine was established by using vibration monitoring compared to rotating machinery standards to give a list of possible components that need to be looked at or changed during engine maintenance.

The two important regions to monitor are at steady state (cruise) flight conditions and at start up and shutdown of engine.

\subsection{Vibration Sensors}

There are three major types of sensors, which may be used to measure machine vibrations and they may be classified by what they measure: displacement, velocity, or acceleration.

Piezoelectric accelerometers are for measuring the vibrations to get an indication of the machine's condition. Typical accelerometers have high natural frequencies of about $25000 \mathrm{~Hz}$ which is a lot higher than the natural frequencies of gas turbine engines, this creates a wide frequency band for measurements. The advantage of using accelerometers is that they are [11]: convenient, small in size, rugged, good frequency response and can endure relatively high temperatures. For these reasons, the accelerometer is the transducer of choice for the health monitoring system.

The best choice is a tri-axial accelerometers mounted on the bearing housings to measure the movement of the shaft. The accelerometers used should have the following specifications to ensure valuable data: Sensitivity of at least $100 \mathrm{mV} / \mathrm{g}$, Amplitude Range $\pm 30 \mathrm{~g}$, Temperature range $0-350^{\circ} \mathrm{C}$. 
Vibra Metrics' Model 5300 was found to meet the requirements.

\subsection{Start-up and Shutdown Condition}

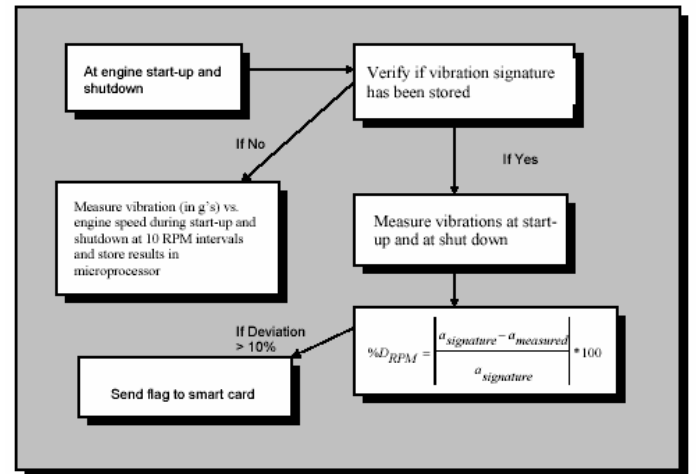

Figure 16. Start-up and shutdown algorithm for vibration monitoring

It is important to measure the vibrations at start-up and shutdown, since the forces that are affecting the engine are transient, and malfunctions may be exhibited which might not be apparent at cruise conditions [12]. Healthy mechanical characteristics of the engine will display a repeatable signature (characteristic frequency spectra) at start-up and shutdown. The first time the engine is run the system will store in the form of a lookup table the acceleration measured by the sensors in intervals of 10 RPM. Then the following times the engine is run, a comparison can be made to the baseline signature and changes greater than $10 \%$ detected will send a flag to the SmartCard. The algorithm for start-up and shut down may be seen in Figure 16.

\subsection{Cruise Condition Vibration Monitoring}

Monitoring the vibration level at cruise condition is very useful to diagnose the engine health by comparing it to limits established by ISO 10816-4 standard for measuring machine vibrations on non-rotating parts for gas turbine engines. The algorithm for steady state condition is shown in Figure 17.

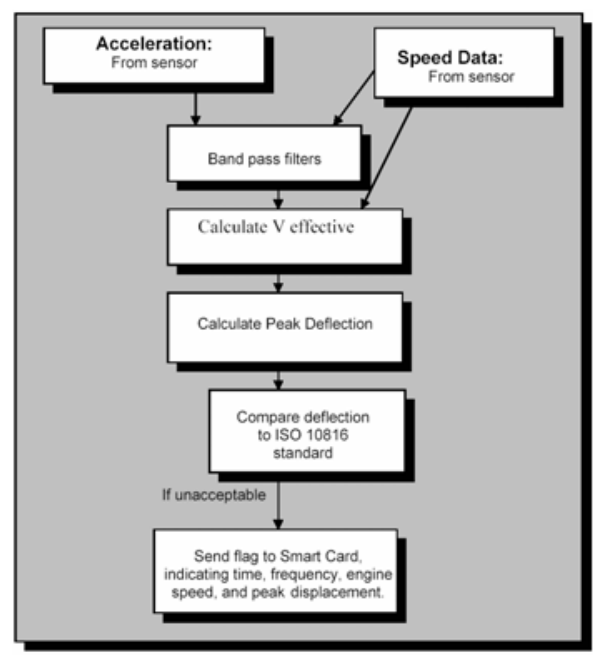

Figure 17. Cruise condition vibration monitoring algorithm

\subsubsection{Data Processing}

During cruise conditions, vibration problems on machinery may be associated with particular harmonic orders or sub harmonics. Table 1 shows the frequencies of interest, the possible causes of vibration and the types of deflections noted.

Where $f_{o}=$ the frequency of the shaft,

Table 1. Vibration characteristics of machines

\begin{tabular}{|l|l|l|}
\multicolumn{1}{|c}{ faults [17] } \\
\hline \multicolumn{1}{|c|}{ Cause of vibration } & \multicolumn{1}{|c|}{ Deflection } & \multicolumn{1}{c|}{ Frequency (Hz) } \\
\hline $\begin{array}{l}\text { Imbalance, incorrect } \\
\text { shaft adjustment }\end{array}$ & $\begin{array}{l}\text { Proportional to the } \\
\text { magnitude of the } \\
\text { imbalance }\end{array}$ & $f_{0}$ \\
\hline $\begin{array}{l}\text { Swinging, alternating } \\
\text { mass forces }\end{array}$ & Variable & $f_{0}, 2 f_{0}$ \\
\hline $\begin{array}{l}\text { Loosening of bearings } \\
\text { Coupling faults, } \\
\text { mechanical looseness }\end{array}$ & Radial & $1 / 2 f_{0}$ \\
\hline $\begin{array}{l}\text { Incorrect bearing } \\
\text { adjustment }\end{array}$ & $\begin{array}{l}\text { The axial deflection } \\
\text { exceeds } 50 \% \text { of the } \\
\text { radial deflection }\end{array}$ & $3 f_{0}$ \\
\hline
\end{tabular}

To be able to get the desired frequencies for vibration measurement, band pass filters will be used.

Once the signals from the accelerometer have been appropriately filtered to measure the desired frequencies, effective velocity (or RMS) is a common means of processing the data [13]. The effective velocity can be calculated using:

$$
V_{\text {eff }}=\sqrt{1 / 2 \sum_{i=0}^{n}\left(\frac{a_{i} * g}{2 \pi f}\right)^{2}}
$$

where: $a_{i}$ is the acceleration in g's

$g$ is gravitational acceleration in $\mathrm{m} / \mathrm{s}^{2}$

$f$ is the frequency of rotation in $\mathrm{Hz}$

An important point to consider is the sampling frequency that will be used to measure the variations in acceleration. Nyquist's criterion states that it is impor- 
tant to take at least two measurements per cycle to get a valid effective velocity of vibration and also to avoid aliasing [14]. However, vibration standards state that 10 times the highest frequency measured should be used [15]. Since engine rotational speed at steady state is less than 4000 RPM (about 70Hz), and also because the fastest frequency measured is $3 f_{\mathrm{o}}(210 \mathrm{~Hz})$, taking measurements at a sampling time of $2.1 \mathrm{kHz}$ within the microprocessor will give good results.

It has been decided that the data acquisition system will take $10 \mathrm{~s}$ measurements at $10 \mathrm{~min}$ intervals, therefore the number of measurements taken to calculate the effective velocity can be found:

$$
\text { \#measurements }=2.1 * 10^{3}(1 / \mathrm{s}) * 10 s=21000
$$

The next step is to change the effective velocity into peak deflection. A graph used for this conversion can be found in reference 15 . This is then compared to established ISO 10816-4 standard for measuring machine vibrations on non-rotating parts for gas turbine engines [16].

\subsubsection{Comparison to ISO Standard}

Now that the vibration is known in terms of peak deflection it is possible to relate it to the possible mechanical wear of the parts of the turbine by comparing the peak deflection to the limits established by ISO 10816-4 standard [16]. The standards present the various regions of probable machine condition. Where the individual regions of the diagram indicate:

I) Dangerous to operate, the turbine must be stopped immediately.

II) The turbine is in a condition that immediately precedes failure.

III) The turbine is damaged.

IV) Slight defects have occurred.

V) The turbine is perfectly faultless.

Equations may be developed to compare the measured deflection to the limit deflection of a curve at a certain engine speed.

For Zone V:

$$
D_{\text {limit }}[\mu m]=10^{-.3116 \log (N[R P M])+2.0108}
$$

For $100<\mathrm{N}<2000 \mathrm{RPM}$

$$
D_{\lim i t}[\mu \mathrm{m}]=10^{-1.818 \log (N[R P M])+7.0237}
$$

For $2000<\mathrm{N}<10000$ RPM

Similar equations may be developed for the other zones.

Then with a simple programming code the appropriate region of vibration may be found as follows:

$\mathrm{D}_{\text {limit I }}-\mathrm{D}_{\text {measured }}=\Delta \mathrm{D}$

If $\Delta \mathrm{D}$ is positive then Zone I

Else

$\mathrm{D}_{\text {limit II }}-\mathrm{D}_{\text {measured }}=\Delta \mathrm{D}$

If $\Delta \mathrm{D}$ is positive then Zone II

Else
And so forth....

\subsubsection{Signal to SmartCard}

The final step is to send a message to the SmartCard if the vibration is found to be in a region other than Zone I. If a message needs to be sent to the card, the following data will be recorded: Zone number, Engine Speed (RPM), Peak Displacement (mm), Frequency of vibration $(\mathrm{Hz})$, Sensor \# and axis, Time of event.

With this information retrieved at landing from the SmartCard, and using Table 1, the possible causes of wear may be determined and appropriate maintenance may be done.

\section{Data Display Software}

The system requires the "continuous" (high sample rate) on-board monitoring of parameters. Even though data is collected at a sufficiently high sampling rate during operation, not all data is stored for further analysis. The objective of the software is that a single value (e.g., mean value, peak value, or total usage) is transmitted to the SmartCard for ground personnel to use during maintenance, in order to detect unusual events general trends over the lifetime of the engine (Figure 18).

The software is programmed to run under the Windows environment. The tools used to write the code is MFC (Microsoft Foundation Classes) which is a predefined library of $\mathrm{C}++$ classes by Microsoft. The main features that control the data from the SmartCard are: card load, back, next. The "next" and "back" functions are required to allow the user to navigate the other days' information. They can be found on either the menu or the toolbar

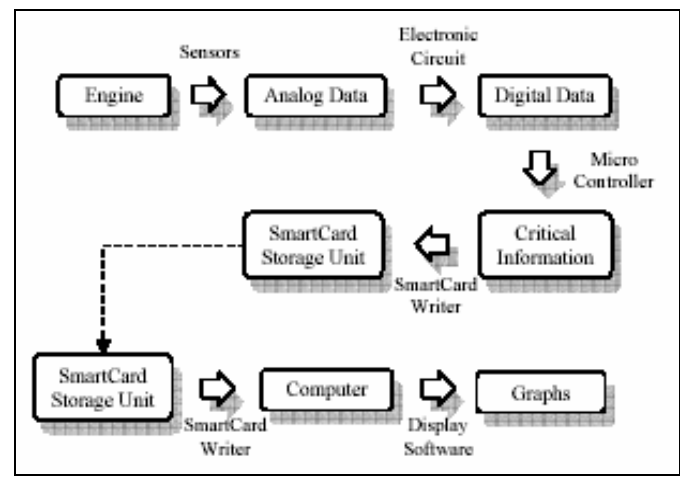

Figure 18. Data processing

The user is required to enter a password in order to keep the information confidential. Once the correct login has been entered, the software will start taking the information from the SmartCard. The functions used to control the SmartCard come from predefined source files provided by the manufacturer of the SmartCard 
reader/writer. The software will enable the detection of any unusual response and output the respective error message.

If there are no errors, the data will be recorded by the software, which will classify the information by date in a bar chart manner. A dashed line in the graph demarcates the threshold value line.

\section{Conclusion}

This paper presented the findings of the capstone design project team assigned to design a comprehensive cost effective monitoring system for gas turbine engines. This system was to be implemented using SmartCard technology. The achievements of this Capstone project have been: Independent validation of GSP-10 software, full simulation of the SR-30 mini-lab gas turbine, a comprehensive aerodynamic component monitoring system, a comprehensive mechanical system monitoring scheme and a GUI for receiving data from the SmartCard.

Accurate monitoring algorithms require accurate predictive models. With 0 -D modeling of a gas turbine, being an integral part of developing a health monitoring system, GSP proved to provide realistic results. The accuracy of the simulation is strongly dependant on the use of precise engine characteristic performance maps. When experimentally obtained performance maps cannot be obtained in open source, the maps must be obtained through aerodynamics and loss correlations.

Other models used in the system require material strength data (Goodman and Larsen Miller for example), as well as geometric parameters used for dynamic vibration modeling of the mechanical systems. This combination of aerodynamic modeling and mechanical performance testing of the engine is what makes the low cost data acquisition system robust. This being said, the predicting ability of the system directly depends on the accuracy of this data.

\section{References}

[1] SAE ARP 1587. "Aircraft gas turbine engine monitoring system guide." Aerospace Recommended Practice, April 1981.

[2] Mini-Lab SR-30 Gas Turbine Engine Document, Turbine Technologies, Chetek, WI, 2004.

[3] T. Witkowski, S. White, C. Ortiz Dueñas, P. Strykowski, T. Simon, "Characterizing the Performance of the SR-30 Turbojet Engine," Proceedings of the 2003 American Society for Engineering Education Annual Conference \& Exposition," American Society for Engineering Education, Washington, DC, 2003.

[4] Moustapha, S. H.; Kacker, S. C.; Tremblay, B.; "An Improved Incidence Losses Prediction Method for
Turbine Airfoils" Journal of Turbomachinery, ASME 89GT-284, Vol 112, April 1990, PP. 267-276.

[5] H. W. Oh, E. S. Yoon, M. K. Chung, "An Optimum Set of Loss Models for Performance Prediction of Centrifugal Compressors," Proc Instn Mech Engrs, Vol 211, Part A, April 1997, PP. 331-338.

[6] SAE Aerospace Recommended Practice, "ARP1217 Instrumentation Requirements for Turboshaft Engine Performance Measurments," Society of Automotive Engineers, Inc. 1997.

[7] Boyce, Meherwan P., Gas Turbine Engine Handbook $: 2^{\text {nd }}$ ed. Gulf Profesional Publishing, 2002. 411-431, TJ778-B67

[8] Figliola, R., Beasley, D. Theory and Design for Mechanical Measurements, John Wiley \& Sons Inc., New York, 2000, pp. 536.

[9] Hardin, Howell, Mirilovich, Hartranft, Schreder "A Gas Turbine Condition Monitoring System", Naval Engineers Journal. 1995, v. 107, n. 6, p. 23, 12 p.

[10] Rao, S.R., Mechanical Vibrations, Pearson Education Inc., New Jersey, 2004, p786, pp. 1078.

[11] Banks, J. C.; Brought, M. S., Byington, C. S. "Vibration sensor configuration optimization for the AV-8B F402-RR-408 engine." (2002) IEEE Aerospace Conference Proceedings, Cat. No.02TH8593, vol. 6

[12] Phillips, J. N., Levine, P., Holm, S. "Optimization of Gas Turbine Plant Operations using a total Condition Monitoring System," Fern Engineering, 20 p.

[13] Wallace, R. H., Understanding and Measuring Vibration, Wykeham Inc, London, 1970, pp. 148.

[14] Wowk, V., Machinery vibration: measurement and analysis, McGraw-Hill, New York, 1991, pp. 358.

[15] Wilson, R.J. “The Design and Development of an Acquisition System and a Computerized Processing Package for the Study of Machinery Vibrations," Thesis in the Faculty of Engineering, Concordia University, 1977, pp 80.

[16] ISO 10816-4 standard for measuring machine vibrations on non-rotating parts for gas turbine engines

[17] Roemer, Michael, Atkinson, Ben "Real-time Health Monitoring and Diagnostics for Gas Turbine Engines", P/PM Technology 1998 , v. 11, n. 5,. 